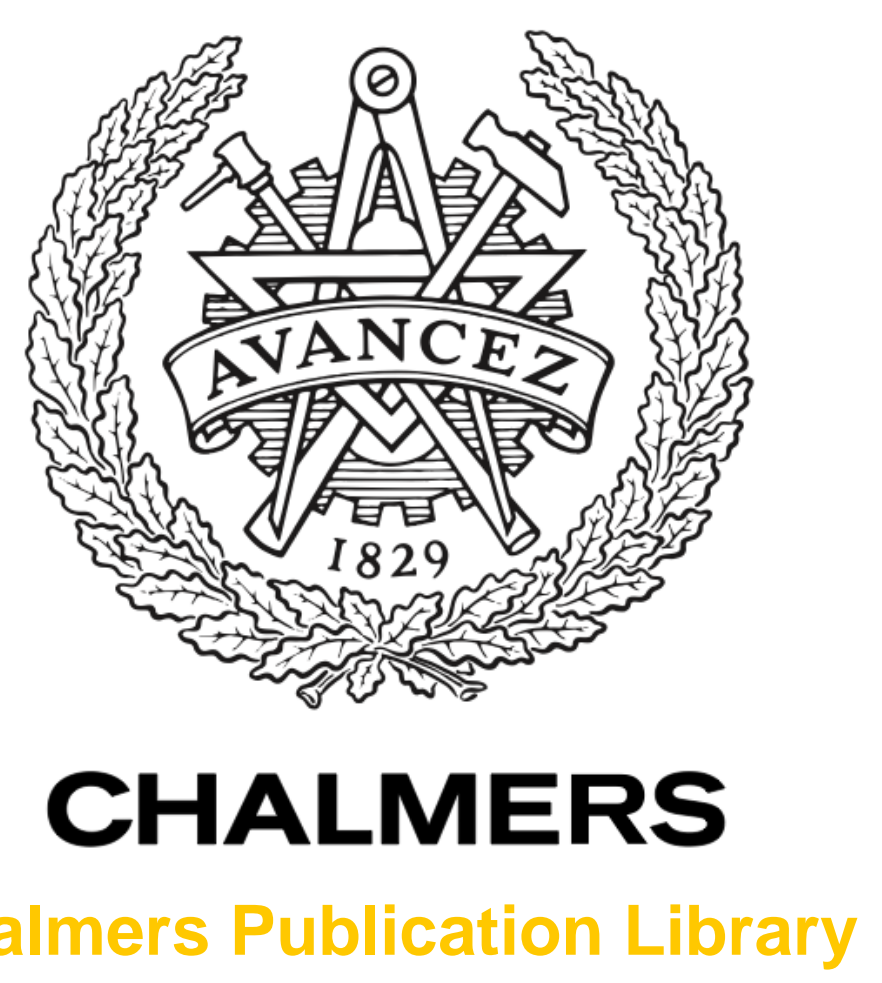

Chalmers Publication Library

Finite Block-Length Analysis of Spectrum Sharing Networks

This document has been downloaded from Chalmers Publication Library $(\mathrm{CPL})$. It is the author's version of a work that was accepted for publication in:

IEEE International Conference on Communications, ICC 2015, London, United Kingdom, 812 June 2015 (ISSN: 1550-3607)

Citation for the published paper:

Makki, B. ; Svensson, T. ; Zorzi, M. (2015) "Finite Block-Length Analysis of Spectrum Sharing Networks". IEEE International Conference on Communications, ICC 2015, London, United Kingdom, 8-12 June 2015 pp. 7665-7670.

http://dx.doi.org/10.1109/ICC.2015.7249552

Downloaded from: http://publications.lib.chalmers.se/publication/204248

Notice: Changes introduced as a result of publishing processes such as copy-editing and formatting may not be reflected in this document. For a definitive version of this work, please refer to the published source. Please note that access to the published version might require a subscription.

Chalmers Publication Library (CPL) offers the possibility of retrieving research publications produced at Chalmers University of Technology. It covers all types of publications: articles, dissertations, licentiate theses, masters theses, conference papers, reports etc. Since 2006 it is the official tool for Chalmers official publication statistics. To ensure that Chalmers research results are disseminated as widely as possible, an Open Access Policy has been adopted.

The CPL service is administrated and maintained by Chalmers Library. 


\title{
Finite Block-Length Analysis of Spectrum Sharing Networks
}

\author{
Behrooz Makki*, Tommy Svensson*, Michele Zorzi ${ }^{\dagger}$ \\ *Department of Signals and Systems, Chalmers University of Technology, Gothenburg, Sweden \\ \{behrooz.makki, tommy.svensson\}@ chalmers.se \\ ${ }^{\dagger}$ Department of Information Engineering, University of Padova, Padova, Italy, \\ zorzi@dei.unipd.it
}

\begin{abstract}
This paper studies the throughput of spectrum sharing networks utilizing rate adaptation. We use some recent results on the achievable rates of finite block-length codes to analyze the secondary user (SU) throughput with a constraint on the primary user (PU) codeword drop probability. With codewords of finite length, we derive closed-form expressions for the SU activation probability and throughput. The results are obtained in different scenarios with no channel state information at the $\mathrm{SU}$ transmitter. As demonstrated numerically and analytically, using finite-length codewords there is considerable potential for the data transmission of unlicensed secondary users under different quality-of-service requirements of the licensed primary users.
\end{abstract}

\section{INTRODUCTION}

Spectrum is a scarce and valuable resource in today's wireless communication networks; with the ever-increasing number of wireless devices such as smart phones, there is growing demand for spectrum resources. This point has led to complaints about spectrum shortage which is expected to grow even more in the future. To tackle the spectrum shortage problem, several solutions have been proposed, with spectrum sharing being among the most promising ones [1]-[19].

In general, the goal of a spectrum sharing scheme is to alleviate the spectrum scarcity problem by allowing unlicensed secondary users (SUs) to access the spectrum that is allocated to licensed primary users (PUs) under the condition of preserving the PUs quality-of-service requirements. There are two approaches to exploit the idea of spectrum sharing, namely, interference-avoiding and simultaneous transmission. The interference-avoiding (interweave) paradigm [19], [20] refers to the scheme where, provided that the SU transmitter can sense the temporal, spatial or spectral gaps of the PU resources, it can adjust its transmission parameters to fill these white spaces. In the simultaneous transmission (underlay) technique, on the other hand, a SU can simultaneously coexist with a PU as long as the PU quality-of-service constraints are satisfied [1]-[18]. In this paper, we concentrate on the simultaneous transmission paradigm of spectrum sharing networks.

The performance of spectrum sharing networks has been studied in various papers, e.g., [2]-[18]. Particularly, considering perfect [2], [5]-[11] and no/imperfect channel state information (CSI) [2]-[5], [12]-[14] at the SU transmitter, the ergodic [2]-[14], the delay-limited [8] and the outage capacity [3], [11] of spectrum sharing networks were investigated,

This work was supported in part by the Swedish Governmental Agency for Innovation Systems (VINNOVA) within the VINN Excellence Center Chase. and results were obtained in the cases with a PU received signal-to-interference-and-noise ratio (SINR) [6], a PU outage probability [11]-[14] and a PU average [2], [4]-[10] or peak interference [3]-[8] constraint. Also, [15]-[18] study the expected throughput [15], [16], the outage probability/throughput [17] and the average rate [18] of the automatic repeat request (ARQ)-based spectrum sharing networks with imperfect CSI.

As a common point, the results of [2]-[18] (and many other papers) are obtained under the assumption of asymptotically long codewords where the instantaneous achievable rate of a user is given by $\log (1+x)$ with $x$ standing for the user instantaneous received SINR. On the other hand, in many applications, such as vehicle-to-vehicle and vehicle-to-infrastructure communications for traffic efficiency/safety or real-time video processing for augmented reality, the codewords are required to be short (in the order of $\sim 100$ channel uses) [21]-[24]. Thus, it is interesting to study the performance of spectrum sharing networks in the presence of finite-length codewords.

In this paper, we study the data transmission efficiency of spectrum sharing networks utilizing codewords of finite length. We use the recent results of [25]-[27] on the achievable rates of finite block-length codes to analyze the system performance. With a constraint on the PU codeword drop probability (DP), we maximize the SU throughput in the cases with no CSI at the SU transmitter. We derive closed-form expressions for the SU activation probability (Lemma 1 and Eq. (19)) and throughput under different PU DPs (Lemma 2). Moreover, we maximize the no-CSI throughput of the SU via rate adaptation.

The numerical and the analytical results demonstrate that, with codewords of finite length, remarkable throughput improvements can be achieved in spectrum sharing networks, if the transmission rates are properly adapted. Moreover, for different PU DP constraints, there are finite optimal SU and PU transmission powers such that the SU DP-limited throughput is maximized (Figs. 2, 3). Finally, for different SU/PU transmission powers and PU DP constraints, the SU throughput is considerably affected by the length of the short codewords. However, for sufficiently long codewords, the throughput degradation due to the finite length of the codewords is negligible (Fig. 4).

\section{System Model}

Consider a spectrum sharing network where a primary user and a secondary user share the same narrow-band frequency 
with normalized bandwidth $W=1$. Let $H_{\mathrm{pp}}, H_{\mathrm{sp}}, H_{\mathrm{ps}}$ and $H_{\mathrm{ss}}$ be the instantaneous fading channel coefficients in the PU-PU, the SU-PU, the PU-SU and the SU-SU links, respectively. Moreover, we define $G_{\mathrm{pp}} \triangleq\left|H_{\mathrm{pp}}\right|^{2}, G_{\mathrm{sp}} \triangleq\left|H_{\mathrm{sp}}\right|^{2}, G_{\mathrm{ps}} \triangleq$ $\left|H_{\mathrm{ps}}\right|^{2}$ and $G_{\mathrm{ss}} \triangleq\left|H_{\mathrm{ss}}\right|^{2}$ which are denoted as channel gains in the following. In this way, the channel outputs are given by

$$
\begin{aligned}
& \left\{\begin{array}{c}
Y_{\mathrm{p}}=\sqrt{T_{\mathrm{p}}} X_{\mathrm{p}} H_{\mathrm{pp}}+\sqrt{T_{\mathrm{s}}} X_{\mathrm{s}} H_{\mathrm{sp}}+Z_{\mathrm{p}} \\
Y_{\mathrm{s}}=\sqrt{T_{\mathrm{s}}} X_{\mathrm{s}} H_{\mathrm{ss}}+\sqrt{T_{\mathrm{p}}} X_{\mathrm{p}} H_{\mathrm{ps}}+Z_{\mathrm{s}}
\end{array}\right. \\
& E\left[\left|X_{\mathrm{p}}\right|^{2}\right]=1, E\left[\left|X_{\mathrm{s}}\right|^{2}\right]=1,
\end{aligned}
$$

where $X_{\mathrm{p}}$ and $X_{\mathrm{s}}$ are the PU and the SU unit-variance input messages, respectively, and $Y_{\mathrm{p}}$ and $Y_{\mathrm{s}}$ denote their corresponding outputs. Also, $T_{\mathrm{p}}$ and $T_{\mathrm{s}}$ represent the PU and the SU input powers, respectively, $E[$.] is the expectation operator and the complex white Gaussian noises $Z_{\mathrm{p}}$ and $Z_{\mathrm{s}}$ added at the PU and $\mathrm{SU}$ receivers are supposed to have distributions $\mathcal{C N}(0,1)$.

The system performance is studied in block-fading conditions, e.g., [1]-[18], [23]-[27], where the channel coefficients remain constant during the channel coherence time, and then change to other values according to the fading probability density function (PDF). The PDF and the cumulative distribution function $(\mathrm{CDF})$ of a random variable $X$ are represented by $f_{X}$ and $F_{X}$, respectively. In harmony with [1]-[11], [13], [14], [17], [18], we assume that there is a feedback link from the $\mathrm{PU}$ or a band manager to the SU, such that in each slot the $\mathrm{SU}$ is informed whether it can become active or not. That is, in each slot the PU permits the SU data transmission, if the PU SINR is so high that its DP constraint is satisfied. In each slot, the receivers are assumed to know their received SINR, which is an acceptable assumption in block-fading conditions [1]-[18], [23]-[27]. Then, the system performance is studied in the cases where the SU transmitter is provided with no information about the SU received SINR. Indeed, performance analysis in the cases with imperfect CSI at the transmitters is an interesting extension of the paper. The results are obtained for Rayleigh fading channels where the channel gains PDFs are given by $f_{G_{\mathrm{pp}}}(x)=\lambda_{\mathrm{pp}} e^{-\lambda_{\mathrm{pp}} x}, f_{G_{\mathrm{sp}}}(x)=\lambda_{\mathrm{sp}} e^{-\lambda_{\mathrm{sp}} x}$, $f_{G_{\mathrm{ps}}}(x)=\lambda_{\mathrm{ps}} e^{-\lambda_{\mathrm{ps}} x}$ and $f_{G_{\mathrm{ss}}}(x)=\lambda_{\mathrm{ss}} e^{-\lambda_{\mathrm{ss}} x}$ with $\lambda_{\mathrm{pp}}, \lambda_{\mathrm{sp}}, \lambda_{\mathrm{ps}}$ and $\lambda_{\mathrm{ss}}$ representing the fading parameters. Under these assumptions, the instantaneous received SINR at the PU and the $\mathrm{SU}$ receivers are given by

$$
\phi_{\mathrm{p}}=\frac{T_{\mathrm{p}} G_{\mathrm{pp}}}{1+T_{\mathrm{s}} G_{\mathrm{sp}}}
$$

and

$$
\phi_{\mathrm{s}}=\frac{T_{\mathrm{s}} G_{\mathrm{ss}}}{1+T_{\mathrm{p}} G_{\mathrm{ps}}}
$$

respectively, and their CDFs are obtained as

$$
\begin{aligned}
& F_{\phi_{\mathrm{p}}}(x)=\operatorname{Pr}\left(\frac{T_{\mathrm{p}} G_{\mathrm{pp}}}{1+T_{\mathrm{s}} G_{\mathrm{sp}}} \leq x\right) \\
& =\int_{0}^{\infty} \lambda_{\mathrm{sp}} e^{-\lambda_{\mathrm{sp}} t} \operatorname{Pr}\left(G_{\mathrm{pp}} \leq \frac{x}{T_{\mathrm{p}}}\left(1+T_{\mathrm{s}} t\right)\right) \mathrm{d} t=1-\frac{e^{-\frac{\lambda_{\mathrm{pp}} x}{T_{\mathrm{p}}}}}{1+\frac{\lambda_{\mathrm{pp}} T_{\mathrm{s}}}{\lambda_{\mathrm{sp}} T_{\mathrm{p}}}} \\
& F_{\phi_{\mathrm{s}}}(x)=1-\frac{e^{-\frac{\lambda_{\mathrm{ss}} x}{T_{\mathrm{s}}}}}{1+\frac{\lambda_{\mathrm{ss}} T_{\mathrm{p}}}{\lambda_{\mathrm{ps}} T_{\mathrm{s}}}}
\end{aligned}
$$

\section{PROBLEM FORMULATION}

We consider a DP constraint for the PU. In each slot, the PU checks whether the channel conditions are good enough so that its DP constraint is satisfied. Then, the PU informs the SU transmitter whether it can send messages or not. Upon receiving this permission, the SU encodes $K_{\mathrm{s}}$ information nats into a codeword of length $L$ and rate $R_{\mathrm{s}}=\frac{K_{\mathrm{s}}}{L}$ natsper-channel-use (npcu), and sends the codeword towards its corresponding receiver. In this way, the optimization problem of the paper is rephrased as

$$
\begin{aligned}
& \max \eta_{\mathrm{s}} \\
& \text { subject to } \Theta_{\mathrm{p}} \leq \theta_{\mathrm{p}},
\end{aligned}
$$

where $\eta_{\mathrm{s}}$ (in npcu) denotes the SU throughput, $\Theta_{\mathrm{p}}$ is the PU DP and $\theta_{\mathrm{p}}$ represents the PU DP constraint. In (5), the optimization can be on different rate and/or power terms of the SU, as long as the other rate/power values are given and the SU throughput and the PU DP are expressed in closedform. Specifically, in the results of Section V we optimize the SU throughput with respect to, e.g., the SU power.

In Section IV, we study (5). First, we derive closed-form expressions for the SU throughput and the PU DP. Then, we use the expressions to analyze the system performance in different conditions. Indeed, to find the expressions, we need to implement approximation techniques. However, as demonstrated in the following, the final conclusions are in harmony with the numerical simulations with high accuracy.

We first review some of the results of [25]-[27] on the achievable rates of the finite-block length codes as follows.

On the achievable rates of the finite-length codes [25]-[27]: Define an $(L, N, T, \delta)$ code as the collection of

- An encoder $\Upsilon:\{1, \ldots, N\} \mapsto \mathcal{C}^{L}$ which maps the message $n \in\{1, \ldots, N\}$ into a length- $L$ codeword $x_{n} \in\left\{x_{1}, \ldots, x_{N}\right\}$ satisfying the power constraint

$$
\frac{1}{L}\left\|x_{j}\right\|^{2} \leq T, \forall j
$$

- A decoder $\Lambda: \mathcal{C}^{L} \mapsto\{1, \ldots, N\}$ satisfying the maximum error probability constraint

$$
\max _{\forall j} \operatorname{Pr}(\Lambda(y) \neq J \mid J=j) \leq \delta
$$

with $y$ denoting the channel output induced by the transmitted codeword.

The maximum achievable rate of the code is defined as

$$
R_{\max }(L, T, \delta)=\sup \left\{\frac{\log N}{L}: \exists(L, N, T, \delta) \text { code }\right\} \text { (npcu). }
$$

Considering non-fading and block-fading conditions with different levels of CSI at the transmitter, [25]-[27] have recently presented a very tight approximation for the maximum achievable rate (8) as

$$
\begin{aligned}
R_{\max }(L, T, \delta) & =\sup \{R: \operatorname{Pr}(\log (1+g T)<R)<\delta\} \\
& -\mathcal{O}\left(\frac{\log L}{L}\right)(\text { npcu }),
\end{aligned}
$$


which, for codes of rate $R$ npcu, leads to the following error probabilities [27, eq. (59)]

$$
\delta_{\text {non-fading }}(L, R, P) \simeq Q\left(\frac{\sqrt{L}(\log (1+g P)-R)}{\sqrt{1-\frac{1}{(1+g P)^{2}}}}\right),
$$

for non-fading channels, and

$$
\delta_{\text {block-fading }}(L, R, P) \simeq E\left[Q\left(\frac{\sqrt{L}(\log (1+g P)-R)}{\sqrt{1-\frac{1}{(1+g P)^{2}}}}\right)\right],
$$

for block-fading channels. Here, $g$ is the instantaneous value of the channel gain, $E[$.] denotes the expectation with respect to the channel gain $g$ and $U(x)=\mathcal{O}(V(x)), x \rightarrow \infty$ is defined as $\lim _{x \rightarrow \infty} \sup \left|\frac{U(x)}{V(x)}\right|<\infty$. Moreover, $Q(x)=\frac{1}{\sqrt{2 \pi}} \int_{x}^{\infty} e^{-\frac{t^{2}}{2}} \mathrm{~d} t$ represents the Gaussian $Q$-function. Since the approximations (10)-(11) have been shown to be very tight for sufficiently large values of $L$ [25]-[27], for simplicity we will assume that they are exact in the following. This is accurate because the practical codes of interest for, e.g., vehicle-to-vehicle communication have been suggested to be in the range of 100-300 channel uses, e.g., see [21]; this is the range of the codewords length for which (10)-(11) are reasonably tight [27]. Also, the error functions in (10)-(11) are the best available closed-form expressions for the performance analysis of finite-length codes.

\section{Throughput Analysis}

In this section, we study the SU throughput in DP-limited conditions. First, we find the PU DP which determines the activation probability of the SU. Then, we analyze the SU throughput for different conditions.

Assuming perfect CSI at the PU receiver, if the SU becomes active, the PU instantaneous error probability (conditional on the fading channel state) is given by (10). Thus, in each slot with an instantaneous SINR realization $\phi_{\mathrm{p}}$, the PU receiver allows the SU transmitter to transmit messages iff

$$
Q\left(\frac{\sqrt{L}\left(\log \left(1+\phi_{\mathrm{p}}\right)-R_{\mathrm{p}}\right)}{\sqrt{1-\frac{1}{\left(1+\phi_{\mathrm{p}}\right)^{2}}}}\right) \leq \theta_{\mathrm{p}}
$$

Here, $R_{\mathrm{p}}=\frac{K_{\mathrm{p}}}{L}$ denotes the PU data rate, with $K_{\mathrm{p}}$ being the number of information nats per PU codeword, and (12) follows from (2) and $(10)^{1}$. That is, in each slot the PU checks whether the instantaneous channel conditions are such that its interference-affected DP satisfies a given quality-of-service requirement. Consequently, the SU activation probability, i.e., the average fraction of time slots where it is possible for the SU to send messages, is obtained by

$\Psi_{\mathrm{s}}=\operatorname{Pr}\left(Q\left(\frac{\sqrt{L}\left(\log \left(1+\phi_{\mathrm{p}}\right)-R_{\mathrm{p}}\right)}{\sqrt{1-\frac{1}{\left(1+\phi_{\mathrm{p}}\right)^{2}}}}\right) \leq \theta_{\mathrm{p}}\right)=1-F_{\phi_{\mathrm{p}}}\left(\xi_{\mathrm{p}}\right)$,

${ }^{1}$ For simplicity, we assume the SU and the PU to have codewords of the same length. It is straightforward to extend the results to the cases with different codeword lengths for the SU and the PU. where

$$
\xi_{\mathrm{p}} \triangleq \arg _{x}\left\{\frac{\sqrt{L}\left(\log (1+x)-R_{\mathrm{p}}\right)}{\sqrt{1-\frac{1}{(1+x)^{2}}}}=Q^{-1}\left(\theta_{\mathrm{p}}\right)\right\} .
$$

In (13) and (14), we have used the fact that the $Q$-function and

$$
W(x)=\frac{\sqrt{L}\left(\log (1+x)-R_{\mathrm{p}}\right)}{\sqrt{1-\frac{1}{(1+x)^{2}}}}
$$

are decreasing and increasing functions of $x$, respectively. Also, $Q^{-1}($.$) represents the inverse Q$-function.

Due to the monotonicity of the $Q(x)$ and $W(x)$ functions, it is straightforward to show that $\xi_{\mathrm{p}}$ will be unique for every given values of $R_{\mathrm{p}}, T_{\mathrm{p}}, T_{\mathrm{s}}$. Unfortunately, there is no general closed-form solution for $\xi_{\mathrm{p}}$. Therefore, we propose approximate solutions of (14) as follows.

Lemma 1: The SU activation probability is obtained as $\Psi_{\mathrm{s}}=\frac{e^{-\frac{\lambda_{\mathrm{pq}} \xi_{\mathrm{p}}}{T_{\mathrm{p}}}}}{1+\frac{\lambda_{\mathrm{p}} T_{\mathrm{s}}}{\lambda_{\mathrm{sp}} T_{\mathrm{p}}} \xi_{\mathrm{p}}}$ where $\xi_{\mathrm{p}}$ is approximately given by

$$
\xi_{\mathrm{p}}=e^{\left(\frac{Q^{-1}\left(\theta_{\mathrm{p}}\right)}{\sqrt{L}}+R_{\mathrm{p}}\right)}-1 .
$$

Proof. Removing the denominator in (14), which is a good approximation for moderate/large values of $x$, we have

$$
\xi_{\mathrm{p}} \simeq \underset{x}{\arg }\left\{\sqrt{L}\left(\log (1+x)-R_{\mathrm{p}}\right)=Q^{-1}\left(\theta_{\mathrm{p}}\right)\right\},
$$

which leads to (16).

It is interesting to note that with an infinitely long code, i.e., letting $L \rightarrow \infty$, we have $\Psi_{\mathrm{s}}=\operatorname{Pr}\left(\log \left(1+\phi_{\mathrm{p}}\right) \geq\right.$ $\left.R_{\mathrm{p}}\right)=1-F_{\phi_{\mathrm{p}}}\left(e^{R_{\mathrm{p}}}-1\right)$, and in each slot the PU knows with certainty whether it can decode its corresponding message correctly. Thus, the finite length of the codewords reduces the SU activation probability by $\Delta \simeq\left(F_{\phi_{\mathrm{p}}}\left(e^{\left(\frac{Q^{-1}\left(\theta_{\mathrm{p}}\right)}{\sqrt{L}}+R_{\mathrm{p}}\right)}-1\right)-\right.$ $\left.F_{\phi_{\mathrm{p}}}\left(e^{R_{\mathrm{p}}}-1\right)\right)$.

In addition to the lemma, we can use the approximations $\log (1+x) \simeq x$ and $\sqrt{1-\frac{1}{(1+x)^{2}}} \simeq \sqrt{2 x}$ for small values of $x$, to rewrite (14) as the solution of

$$
\begin{aligned}
& x-R_{\mathrm{p}}=\frac{Q^{-1}\left(\theta_{\mathrm{p}}\right)}{\sqrt{L}} \sqrt{2 x} \\
& \Rightarrow x^{2}-2\left(R_{\mathrm{p}}+\frac{\left(Q^{-1}\left(\theta_{\mathrm{p}}\right)\right)^{2}}{L}\right) x+R_{\mathrm{p}}^{2}=0 .
\end{aligned}
$$

Then, because from (18) we have $x \geq R_{\mathrm{p}}$ for $\theta_{\mathrm{p}} \leq \frac{1}{2}$ (resp. $x<R_{\mathrm{p}}$ for $\left.\theta_{\mathrm{p}}>\frac{1}{2}\right),(18)$ leads to

$\xi_{\mathrm{p}} \simeq$

$\begin{cases}R_{\mathrm{p}}+\frac{\left(Q^{-1}\left(\theta_{\mathrm{p}}\right)\right)^{2}}{L}+\sqrt{\frac{\left(Q^{-1}\left(\theta_{\mathrm{p}}\right)\right)^{2}}{L}\left(2 R_{\mathrm{p}}+\frac{\left(Q^{-1}\left(\theta_{\mathrm{p}}\right)\right)^{2}}{L}\right)} & \theta_{\mathrm{p}} \leq \frac{1}{2} \\ R_{\mathrm{p}}+\frac{\left(Q^{-1}\left(\theta_{\mathrm{p}}\right)\right)^{2}}{L}-\sqrt{\frac{\left(Q^{-1}\left(\theta_{\mathrm{p}}\right)\right)^{2}}{L}\left(2 R_{\mathrm{p}}+\frac{\left(Q^{-1}\left(\theta_{\mathrm{p}}\right)\right)^{2}}{L}\right)} & \theta_{\mathrm{p}}>\frac{1}{2},\end{cases}$

at low SINRs (see Fig. 1 for the tightness of (16) and (19)).

Receiving the free-to-send feedback signal from the PU receiver, the SU sends the message with rate $R_{\mathrm{s}}$ and the codeword is dropped by the SU receiver if it cannot be 
decoded correctly. Thus, the SU throughput, averaged over many codeword transmissions, is given by

$$
\begin{aligned}
& \eta_{\mathrm{s}}^{\mathrm{no}-\mathrm{CSI}}=\Psi_{\mathrm{s}} R_{\mathrm{s}}\left(1-\theta_{\mathrm{s}}^{\mathrm{no}-\mathrm{CSI}}\right), \\
& \theta_{\mathrm{s}}^{\mathrm{no}-\mathrm{CSI}}=E\left[Q\left(\frac{\sqrt{L}\left(\log \left(1+\phi_{\mathrm{s}}\right)-R_{\mathrm{s}}\right)}{\sqrt{1-\frac{1}{\left(1+\phi_{\mathrm{s}}\right)^{2}}}}\right)\right] .
\end{aligned}
$$

Here, $\theta_{\mathrm{s}}^{\text {no-CSI }}$ is the SU DP with codeword rate $R_{\mathrm{s}}$ and no CSI at the transmitter. In order to find (20), we should determine $\theta_{\mathrm{s}}^{\text {no-CSI }}$, which does not have a closed-form expression. The following lemma approximates (20) as follows.

Lemma 2: The no-CSI throughput of the SU is given by $\eta_{\mathrm{s}}^{\mathrm{no}-\mathrm{CSI}}=\Psi_{\mathrm{s}} R_{\mathrm{s}}\left(1-\theta_{\mathrm{s}}^{\text {no-CSI }}\right)$ where $\theta_{\mathrm{s}}^{\mathrm{no}-\mathrm{CSI}}$ is approximated as

$$
\begin{aligned}
& \theta_{\mathrm{s}}^{\text {no-CSI }} \simeq 1-\frac{e^{-\frac{\lambda_{\mathrm{ss}}\left(\alpha_{\mathrm{s}}-\frac{1}{2 \mu_{\mathrm{s}}}\right)}{T_{\mathrm{s}}}}}{1+\frac{\lambda_{\mathrm{ss}} T_{\mathrm{p}}}{\lambda_{\mathrm{ps}} T_{\mathrm{s}}}\left(\alpha_{\mathrm{s}}-\frac{1}{2 \mu_{\mathrm{s}}}\right)} \\
& +\left(\alpha_{\mathrm{s}} \mu_{\mathrm{s}}+\frac{1}{2}\right)\left(\frac{e^{-\frac{\lambda_{\mathrm{ss}}\left(\alpha_{\mathrm{s}}-\frac{1}{2 \mu_{\mathrm{s}}}\right)}{T_{\mathrm{s}}}}}{1+\frac{\lambda_{\mathrm{ss}} T_{\mathrm{p}}}{\lambda_{\mathrm{ps}} T_{\mathrm{s}}}\left(\alpha_{\mathrm{s}}-\frac{1}{2 \mu_{\mathrm{s}}}\right)}-\frac{e^{-\frac{\lambda_{\mathrm{ss}}\left(\alpha_{\mathrm{s}}+\frac{1}{2 \mu_{\mathrm{s}}}\right)}{T_{\mathrm{s}}}}}{1+\frac{\lambda_{\mathrm{ss}} T_{\mathrm{p}}}{\lambda_{\mathrm{ps}} T_{\mathrm{s}}}\left(\alpha_{\mathrm{s}}+\frac{1}{2 \mu_{\mathrm{s}}}\right)}\right) \\
& -\mu_{\mathrm{s}}\left(\left(\alpha_{\mathrm{s}}-\frac{1}{2 \mu_{\mathrm{s}}}\right)\left(\frac{e^{-\frac{\lambda_{\mathrm{ss}}\left(\alpha_{\mathrm{s}}-\frac{1}{2 \mu_{\mathrm{s}}}\right)}{T_{\mathrm{s}}}}}{1+\frac{\lambda_{\mathrm{ss}} T_{\mathrm{p}}}{\lambda_{\mathrm{ps}} T_{\mathrm{s}}}\left(\alpha_{\mathrm{s}}-\frac{1}{2 \mu_{\mathrm{s}}}\right)}\right)\right. \\
& +\left(\alpha_{\mathrm{s}}+\frac{1}{2 \mu_{\mathrm{s}}}\right)\left(\frac{e^{-\frac{\lambda_{\mathrm{ss}}\left(\alpha_{\mathrm{s}}+\frac{1}{2 \mu_{\mathrm{s}}}\right)}{T_{\mathrm{s}}}}}{1+\frac{\lambda_{\mathrm{ss}} T_{\mathrm{p}}}{\lambda_{\mathrm{ps}} T_{\mathrm{s}}}\left(\alpha_{\mathrm{s}}+\frac{1}{2 \mu_{\mathrm{s}}}\right)}\right) \\
& +\frac{\lambda_{\mathrm{ps}} T_{\mathrm{s}} e^{\frac{\lambda_{\mathrm{ps}}}{T_{\mathrm{p}}}} \operatorname{Ei}\left(-\frac{\lambda_{\mathrm{ss}}}{T_{\mathrm{s}}}\left(\alpha_{\mathrm{s}}+\frac{1}{2 \mu_{\mathrm{s}}}\right)-\frac{\lambda_{\mathrm{ps}}}{T_{\mathrm{p}}}\right)}{\lambda_{\mathrm{ss}} T_{\mathrm{p}}} \\
& \left.-\frac{\lambda_{\mathrm{ps}} T_{\mathrm{s}} e^{\frac{\lambda_{\mathrm{ps}}}{T_{\mathrm{p}}}} \operatorname{Ei}\left(-\frac{\lambda_{\mathrm{ss}}}{T_{\mathrm{s}}}\left(\alpha_{\mathrm{s}}-\frac{1}{2 \mu_{\mathrm{s}}}\right)-\frac{\lambda_{\mathrm{ps}}}{T_{\mathrm{p}}}\right)}{\lambda_{\mathrm{ss}} T_{\mathrm{p}}}\right)
\end{aligned}
$$

Here, $\operatorname{Ei}(x)=\int_{-x}^{\infty} \frac{e^{-t}}{t} \mathrm{~d} t$ represents the exponential integral function, $\alpha_{\mathrm{s}} \triangleq e^{R_{\mathrm{s}}}-1$ and $\mu_{\mathrm{s}} \triangleq \sqrt{\frac{L}{2 \pi\left(e^{2 R_{\mathrm{s}}}-1\right)}}$.

Proof. To prove the lemma, we use a linearization technique for the function $Q\left(\frac{\sqrt{L}\left(\log (1+x)-R_{\mathrm{s}}\right)}{\sqrt{1-\frac{1}{(1+x)^{2}}}}\right)$ at point $x=\alpha_{\mathrm{s}}$ to write

$$
\begin{aligned}
& Q\left(\frac{\sqrt{L}\left(\log (1+x)-R_{\mathrm{s}}\right)}{\sqrt{1-\frac{1}{(1+x)^{2}}}}\right) \simeq U_{\mathrm{s}}(x) \\
& U_{\mathrm{s}}(x)=\left\{\begin{array}{cc}
1 & x \leq \alpha_{\mathrm{s}}-\frac{1}{2 \mu_{\mathrm{s}}}, \\
\frac{1}{2}-\mu_{\mathrm{s}}\left(x-\alpha_{\mathrm{s}}\right) & x \in\left(\alpha_{\mathrm{s}}-\frac{1}{2 \mu_{\mathrm{s}}}, \alpha_{\mathrm{s}}+\frac{1}{2 \mu_{\mathrm{s}}}\right), \\
0 & x \geq \alpha_{\mathrm{s}}+\frac{1}{2 \mu_{\mathrm{s}}},
\end{array}\right.
\end{aligned}
$$

with

$$
\mu_{\mathrm{s}}=-\left.\frac{\partial\left(Q\left(\frac{\sqrt{L}\left(\log (1+x)-R_{\mathrm{s}}\right)}{\sqrt{1-\frac{1}{(1+x)^{2}}}}\right)\right)}{\partial x}\right|_{x=\alpha_{\mathrm{s}}}=\sqrt{\frac{L}{2 \pi\left(e^{2 R_{\mathrm{s}}}-1\right)}}
$$

which is found from the derivative of $Q\left(\frac{\sqrt{L}\left(\log (1+x)-R_{\mathrm{s}}\right)}{\sqrt{1-\frac{1}{(1+x)^{2}}}}\right)$ at point $x=\alpha_{\mathrm{s}}$.
Using (20) and (22), the no-CSI DP of the SU is found as

$$
\begin{aligned}
\theta_{\mathrm{s}}^{\mathrm{no}-\mathrm{CSI}}= & \int_{0}^{\infty} f_{\phi_{\mathrm{s}}}(x) Q\left(\frac{\sqrt{L}\left(\log \left(1+\phi_{\mathrm{s}}\right)-R_{\mathrm{s}}\right)}{\sqrt{1-\frac{1}{\left(1+\phi_{\mathrm{s}}\right)^{2}}}}\right) \mathrm{d} x \\
\simeq & F_{\phi_{\mathrm{s}}}\left(\alpha_{\mathrm{s}}-\frac{1}{2 \mu_{\mathrm{s}}}\right)+\left(\alpha_{\mathrm{s}} \mu_{\mathrm{s}}+\frac{1}{2}\right)\left(F_{\phi_{\mathrm{s}}}\left(\alpha_{\mathrm{s}}+\frac{1}{2 \mu_{\mathrm{s}}}\right)\right. \\
& \left.-F_{\phi_{\mathrm{s}}}\left(\alpha_{\mathrm{s}}-\frac{1}{2 \mu_{\mathrm{s}}}\right)\right)-\mu_{\mathrm{s}} \int_{\alpha_{\mathrm{s}}-\frac{1}{2 \mu_{\mathrm{s}}}}^{\alpha_{\mathrm{s}}+\frac{1}{2 \mu_{\mathrm{s}}}} x f_{\phi_{\mathrm{s}}}(x) \mathrm{d} x \\
= & -\frac{e^{-\frac{\lambda_{\mathrm{ss}}\left(\alpha_{\mathrm{s}}-\frac{1}{2 \mu_{\mathrm{s}}}\right)}{T_{\mathrm{s}}}}}{1+\frac{\lambda_{\mathrm{ss}} T_{\mathrm{p}}}{\lambda_{\mathrm{ps}} T_{\mathrm{s}}}\left(\alpha_{\mathrm{s}}-\frac{1}{2 \mu_{\mathrm{s}}}\right)} \\
+ & \left(\alpha_{\mathrm{s}} \mu_{\mathrm{s}}+\frac{1}{2}\right)\left(\frac{e^{-\frac{\lambda_{\mathrm{ss}}\left(\alpha_{\mathrm{s}}-\frac{1}{2 \mu_{\mathrm{s}}}\right)}{T_{\mathrm{s}}}}}{1+\frac{\lambda_{\mathrm{ss}} T_{\mathrm{p}}}{\lambda_{\mathrm{ps}} T_{\mathrm{s}}}\left(\alpha_{\mathrm{s}}-\frac{1}{2 \mu_{\mathrm{s}}}\right)}-\frac{e^{-\frac{\lambda_{\mathrm{ss}}\left(\alpha_{\mathrm{s}}+\frac{1}{2 \mu_{\mathrm{s}}}\right.}{T_{\mathrm{s}}}}}{1+\frac{\lambda_{\mathrm{ss}} T_{\mathrm{p}}}{\lambda_{\mathrm{ps}} T_{\mathrm{s}}}\left(\alpha_{\mathrm{s}}+\frac{1}{2 \mu_{\mathrm{s}}}\right)}\right) \\
- & \mu_{\mathrm{s}}\left(\left(\alpha_{\mathrm{s}}-\frac{1}{2 \mu_{\mathrm{s}}}\right)\left(\frac{e^{-\frac{\lambda_{\mathrm{ss}}\left(\alpha_{\mathrm{s}}-\frac{1}{2 \mu_{\mathrm{s}}}\right.}{T_{\mathrm{s}}}}}{1+\frac{\lambda_{\mathrm{s}} T_{\mathrm{p}}}{\lambda_{\mathrm{s}} T_{\mathrm{s}}}\left(\alpha_{\mathrm{s}}-\frac{1}{2 \mu_{\mathrm{s}}}\right)}\right)\right. \\
- & \left.\left(\alpha_{\mathrm{s}}+\frac{1}{2 \mu_{\mathrm{s}}}\right)\left(\frac{e^{-\frac{\lambda_{\mathrm{ss}}\left(\alpha_{\mathrm{s}}+\frac{1}{2 \mu_{\mathrm{s}}}\right)}{T_{\mathrm{s}}}}}{1+\frac{\lambda_{\mathrm{s}} T_{\mathrm{p}}}{\lambda_{\mathrm{ps}} T_{\mathrm{s}}}\left(\alpha_{\mathrm{s}}+\frac{1}{2 \mu_{\mathrm{s}}}\right)}\right)+\int_{\alpha_{\mathrm{s}}-\frac{1}{2 \mu_{\mathrm{s}}}}^{\alpha_{\mathrm{s}}+\frac{1}{2 \mu_{\mathrm{s}}}} \frac{e^{-\frac{\lambda_{\mathrm{ss}} x}{T_{\mathrm{s}}}}}{1+\frac{\lambda_{\mathrm{ss}} T_{\mathrm{p}}}{\lambda_{\mathrm{ps}} T_{\mathrm{s}}}} \mathrm{d} x\right)
\end{aligned}
$$

which, using the definition of the exponential integral function in the last integration, leads to (21) in the lemma. In (23), the approximation comes from (22) and the last equality is found by (4) and partial integration.

Note that, although the results are obtained for spectrum sharing networks, the same approximation techniques are useful for the finite block-length analysis of single-user networks. Furthermore, using the approximate expressions of the throughput, we can solve (5) numerically or analytically, which is not a difficult task. Finally, to close the section, it is worth noting that with an infinitely long code, i.e., $L \rightarrow \infty$, the SU no-CSI throughput is given by

$$
\begin{aligned}
& \eta_{\mathrm{s}, L \rightarrow \infty}^{\mathrm{no}-\mathrm{CSI}}=\Psi_{\mathrm{s}} R_{\mathrm{s}} \operatorname{Pr}\left(\log \left(1+\phi_{\mathrm{s}}\right) \geq R_{\mathrm{s}}\right) \\
& =\Psi_{\mathrm{s}} R_{\mathrm{s}}\left(1-F_{\phi_{\mathrm{s}}}\left(e^{R_{\mathrm{s}}}-1\right)\right)=\frac{\Psi_{\mathrm{s}} R_{\mathrm{s}} e^{-\frac{\lambda_{\mathrm{ss}}\left(e^{R_{\mathrm{s}}}-1\right)}{T_{\mathrm{s}}}}}{\left.1+\frac{\lambda_{\mathrm{ss}} T_{\mathrm{p}}}{\lambda_{\mathrm{ps}} T_{\mathrm{s}}} e^{R_{\mathrm{s}}}-1\right)},
\end{aligned}
$$

which can be maximized numerically or analytically. For instance, letting $L \rightarrow \infty$ in Lemma 2 , setting the derivative of the throughput with respect to $R_{\mathrm{s}}$ equal to zero and implementing some manipulations, we have

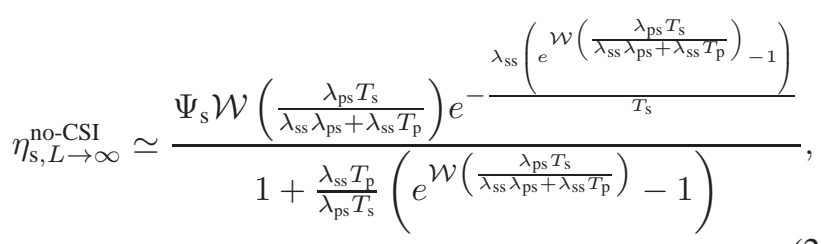

in the optimal case, where $\mathcal{W}$ is the Lambert $\mathrm{W}$ function. In Section V, we validate the accuracy of the approximations proposed in (16)-(25) by comparing them with the corresponding exact values that can be evaluated numerically. 


\section{NUMERICAL RESULTS}

In all figures, we set $\lambda_{\mathrm{ss}}=\lambda_{\mathrm{sp}}=\lambda_{\mathrm{ps}}=\lambda_{\mathrm{pp}}=1$, unless otherwise stated. Thus, as the noise variances are set to $1, T_{\mathrm{p}}$ and $T_{\mathrm{s}}$ (in $\mathrm{dB}, 10 \log _{10} T_{\mathrm{p}}$ and $10 \log _{10} T_{\mathrm{s}}$ ) represent the PU and the SU signal-to-noise ratio (SNR) as well. According to [26], [27], the approximations in (9)-(11) are very tight for sufficiently long codewords, and the tightness increases with the codewords' length. For the numerical results, we consider cases with $L \geq 50$ channel uses, for which the approximation is tight [26], [27], and do not consider shorter codewords. In the meantime, although the approximation is not tight for small $L$ 's and the results should not be fully trusted in that case, we observed the same qualitative conclusions as in the case of $L \geq 50$, when the simulations are run for very short (practically not interesting) codewords (see [26], [27] for more discussions on the tightness of (9)-(11) and [21] for practical codes of interest in, e.g., vehicle-to-vehicle communication).

Setting $R_{\mathrm{p}}=1 \mathrm{npcu}, L=100$ and $\theta_{\mathrm{p}}=10^{-3}$, Fig. 1 shows the SU activation probability given in (13)-(14) and compares the results with the ones in Lemma 1 and (19). As demonstrated in the figure, the approximation method (19) is tight at low SU SINRs, while the tightness decreases with the SU SINR. On the other hand, the approximation approach of Lemma 1, i.e., (16), is very tight for all ranges of SU and PU SINRs. For this reason and also to avoid mixing the effects of different approximations, in the following figures the SU activation probability $\Psi_{\mathrm{s}}$ is calculated numerically, while the same curves (with very high accuracy) are obtained if $\Psi_{\mathrm{s}}$ is derived via Lemma 1 . That is, for all considered scenarios the curves are indistinguishable whether the SU activation probability is determined numerically or via Lemma 1.

In Figs. 2-4, we study the system throughput. Figure 2 verifies the tightness of the approximations derived in Lemma 2. Here, the results are obtained for $L=200, T_{\mathrm{p}}=$ $10,15 \mathrm{~dB}, R_{\mathrm{s}}=R_{\mathrm{p}}=1 \mathrm{npcu}$ and $\theta_{\mathrm{p}}=10^{-2}$. Moreover, shown in Fig. 3 are the maximum SU DP-limited throughput, optimized according to (20), versus the SU transmission power. Here, the simulation results are compared with the ones derived in the cases with asymptotically long codewords, i.e., (24)-(25). Finally, Fig. 4 evaluates the effect of the PU DP constraint, where the SU no-CSI throughput is plotted versus the PU DP constraints for different lengths of the codewords.

According to the figures, the following conclusions can be drawn:

- As seen in Fig. 2, the approximation approach of Lemma 2 is very tight for a broad range of SINRs/parameter settings.

- Figures 2 and 3 indicate that with a PU DP constraint there are finite optimal transmission powers for the SU and the PU such that the SU throughput is maximized. However, the SU DP-limited throughput converges to zero at low and high SINRs. Intuitively, this is because, with a PU DP constraint, at high SINRs (resp. low SINRs) no data transmission is allowed by the PU (resp. the SU achievable rate is small although it is active in many time slots). In harmony with (20), the optimal SU power depends on the codewords length. Moreover, the opti-

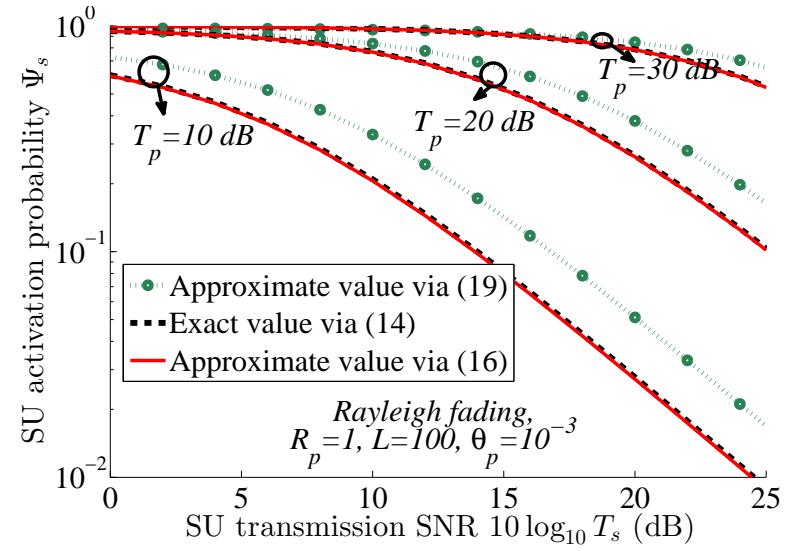

Figure 1. SU activation probability for different SU transmission SNRs $10 \log _{10} T_{\mathrm{s}}(\mathrm{dB})$, Rayleigh fading channels, $L=100, R_{\mathrm{p}}=1 \mathrm{npcu}$, $\theta_{\mathrm{p}}=10^{-3}$

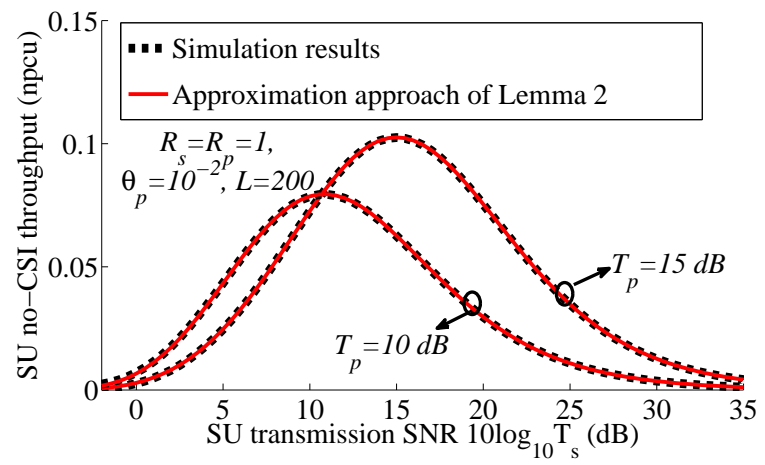

Figure 2. On the tightness of the approximations of Lemma 2, Rayleigh fading channels, no CSI at the SU transmitter, $L=200, R_{\mathrm{p}}=R_{\mathrm{s}}=1 \mathrm{npcu}$, $\theta_{\mathrm{p}}=10^{-2}$

mal SU transmission power maximizing the DP-limited throughput increases with the length of the codewords (Fig. 3).

- For short codewords, the SU DP-limited throughput is remarkably affected by the length of the codewords at hard DP-limited conditions, i.e., when $\theta_{\mathrm{p}}$ is small (Fig. 4). However, the effect of the finite-length codewords decreases when the PU DP constraint is relaxed, i.e., $\theta_{\mathrm{p}}$ increases, and the throughput is less sensitive to the PU DP constraint for long codewords (Fig. 4). Also, the throughput increases with the length of the codewords, as expected. As shown in Fig. 4, the finite length of the codewords leads to considerable SU throughput degradation, for codewords of short length. However, with different SU/PU transmission powers and the considered PU DP constraints, the effect of the codewords length on the SU throughput is negligible for long codewords.

- The tolerance of the primary user, modeled by the DP constraint $\theta_{\mathrm{p}}$, plays a great role in the secondary channel throughput. That is, the more secure the PU qualityof-service requirement, the less the data transmission allowed within the PU activation time slots and the less the throughput achieved at the secondary channel, converging to zero (Fig. 4).

Finally, the results emphasize that, using finite-length codewords and rate adaptation, there is considerable potential for the data transmission of the unlicensed secondary users 


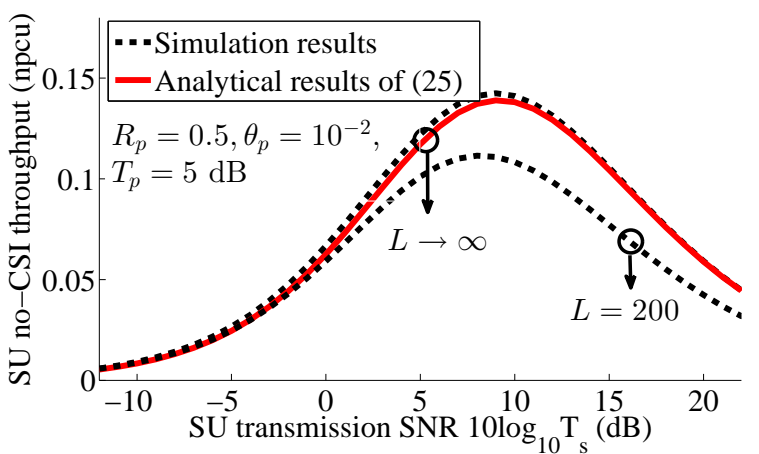

Figure 3. SU maximum throughput for different lengths of the codewords. The black dashed curves are obtained by numerical optimization of (20). Then, the numerical results are compared with the ones derived by (25). Rayleigh fading channels, $R_{\mathrm{p}}=0.5 \mathrm{npcu}, \theta_{\mathrm{p}}=10^{-2}, T_{\mathrm{p}}=5 \mathrm{~dB}$.

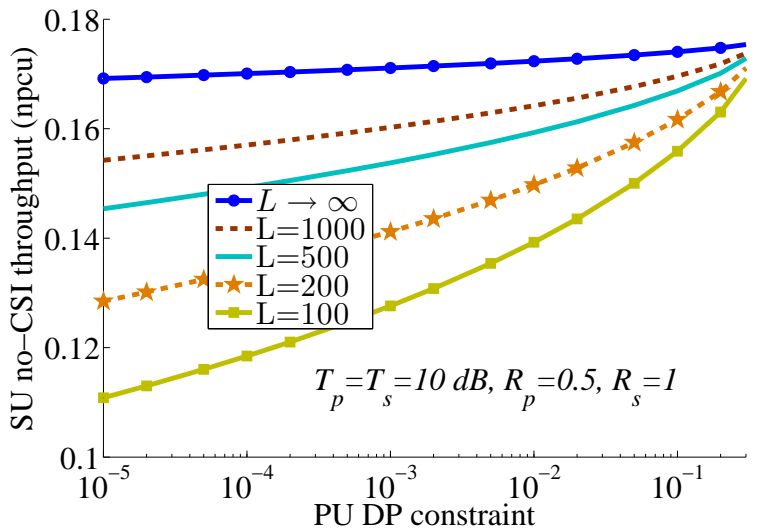

Figure 4. SU no-CSI throughput vs PU DP constraint and different lengths of the codewords. Rayleigh fading channels, $R_{\mathrm{p}}=0.5 \mathrm{npcu}, R_{\mathrm{S}}=1 \mathrm{npcu}$, $T_{\mathrm{p}}=T_{\mathrm{s}}=10 \mathrm{~dB}$.

under different quality-of-service requirements of the licensed primary users.

\section{CONCLUSION}

Considering PU DP-limited conditions, we studied the throughput of spectrum sharing networks with codewords of finite length. The results were obtained in the cases with no-CSI at the SU transmitter. We derived closed-form approximations for the SU throughput and the PU DP. As demonstrated both numerically and analytically, the finite length of the codewords leads to remarkable SU throughput degradation, for codewords of short length. However, with different SU/PU transmission powers and PU DP constraints, the effect of the codewords length on the SU throughput decreases for long codewords. Finally, for different PU DP constraints, there are finite optimal SU and PU transmission powers such that the SU DP-limited throughput is maximized.

\section{REFERENCES}

[1] S. Y. Lien, K. C. Chen, Y. C. Liang, and Y. Lin, "Cognitive radio resource management for future cellular networks," IEEE Wireless Commun., vol. 21, no. 1, pp. 70-79, Feb. 2014.

[2] V. Asghari and S. Aissa, "Resource management in spectrum-sharing cognitive radio broadcast channels: Adaptive time and power allocation," IEEE Trans. Commun., vol. 59, no. 5, pp. 1446-1457, May 2011.

[3] H. A. Suraweera, P. J. Smith, and M. Shafi, "Capacity limits and performance analysis of cognitive radio with imperfect channel knowledge," IEEE Trans. Veh. Technol., vol. 59, no. 4, pp. 1811-1822, May 2010.

[4] L. Musavian and S. Aissa, "Fundamental capacity limits of cognitive radio in fading environments with imperfect channel information," IEEE Trans. Commun., vol. 57, no. 11, pp. 3472-3480, Nov. 2009.
[5] L. Sboui, Z. Rezki, and M. S. Alouini, "Capacity of spectrum sharing cognitive radio systems over Nakagami fading channels at low SNR," in IEEE ICC, June 2013, pp. 5674-5678.

[6] B. Makki and T. Eriksson, "On the ergodic achievable rates of spectrum sharing networks with finite backlogged primary users and an interference indicator signal," IEEE Trans. Wireless Commun., vol. 11, no. 9, pp. 3079-3089, Sept. 2012.

[7] A. Ghasemi and E. S. Sousa, "Fundamental limits of spectrum-sharing in fading environments," IEEE Trans. Wireless Commun., vol. 6, no. 2, pp. 649-658, Feb. 2007.

[8] X. Kang, Y.-C. Liang, A. Nallanathan, H. K. Garg, and R. Zhang, "Optimal power allocation for fading channels in cognitive radio networks: Ergodic capacity and outage capacity," IEEE Trans. Wireless Commun., vol. 8, no. 2, pp. 940-950, Feb. 2009.

[9] C.-X. Wang, X. Hong, H.-H. Chen, and J. Thompson, "On capacity of cognitive radio networks with average interference power constraints," IEEE Trans. Wireless Commun., vol. 8, no. 4, pp. 1620-1625, April 2009.

[10] G. Noh, S. Lim, and D. Hong, "Exact capacity analysis of spectrum sharing systems: Average received-power constraint," IEEE Commun. Lett., vol. 17, no. 5, pp. 884-887, May 2013.

[11] X. Kang, R. Zhang, Y. C. Liang, and H. K. Garg, "Optimal power allocation strategies for fading cognitive radio channels with primary user outage constraint," IEEE J. Sel. Areas Commun., vol. 29, no. 2, pp. 374-383, Feb. 2011

[12] X. Gong, A. Ispas, G. Dartmann, and G. Ascheid, "Outage-constrained power allocation in spectrum sharing systems with partial CSI," IEEE Trans. Commun., vol. 62, no. 2, pp. 452-466, Feb. 2014.

[13] L. Sboui, Z. Rezki, and M. S. Alouini, "A unified framework for the ergodic capacity of spectrum sharing cognitive radio systems," IEEE Trans. Wireless Commun., vol. 12, no. 2, pp. 877-887, Feb. 2013.

[14] Z. Rezki and M. S. Alouini, "Ergodic capacity of cognitive radio under imperfect channel-state information," IEEE Trans. Veh. Technol., vol. 61, no. 5, pp. 2108-2119, June 2012.

[15] R. A. Tannious and A. Nosratinia, "Cognitive radio protocols based on exploiting hybrid ARQ retransmissions," IEEE Trans. Wireless Commun., vol. 9, no. 9, pp. 2833-2841, 2010.

[16] N. Michelusi, P. Popovski, O. Simeone, M. Levorato, and M. Zorzi, "Cognitive access policies under a primary ARQ process via forwardbackward interference cancellation," IEEE J. Sel. Areas Commun., vol. 31, no. 11, pp. 2374-2386, Nov. 2013.

[17] B. Makki, A. Graell i Amat, and T. Eriksson, "HARQ feedback in spectrum sharing networks," IEEE Commun. Lett., vol. 16, no. 9, pp. 1337-1340, Sept. 2012.

[18] B. Makki and T. Eriksson, "On the average rate of HARQ-based quasistatic spectrum sharing networks," IEEE Trans. Wireless Commun., vol. 11, no. 1, pp. 65-77, Jan. 2012.

[19] N. Devroye, M. Vu, and V. Tarokh, "Cognitive radio networks," IEEE Signal Process. Mag., vol. 25, no. 6, pp. 12-23, Nov. 2008.

[20] N. Devroye, P. Mitran, and V. Tarokh, "Achievable rates in cognitive radio channels," IEEE Trans. Inf. Theory, vol. 52, no. 5, pp. 1813-1827, May 2006.

[21] K. Bilstrup, E. Uhlemann, E. G. Strom, and U. Bilstrup, "Evaluation of the IEEE 802.11p MAC method for vehicle-to-vehicle communication," in IEEE VTC Fall, Sept. 2008, pp. 1-5.

[22] EU FP7 INFSO-ICT-317669 METIS project, Deliverable D1.1, "Scenarios, requirements and KPIs for $5 \mathrm{G}$ mobile and wireless system" Tech. Rep., April 2013. Online: https://www.metis2020.com.

[23] B. Makki, T. Svensson, and M. Zorzi, "Finite block-length analysis of the incremental redundancy HARQ," IEEE Wireless Commun. Lett., vol. 3, no. 5, pp. 529-532, Oct. 2014.

[24] — "Green communication via Type-I ARQ: Finite block-length analysis," in IEEE GLOBECOM, Dec. 2014, pp. 2673-2677.

[25] Y. Polyanskiy, H. V. Poor, and S. Verdu, "Channel coding rate in the finite blocklength regime," IEEE Trans. Inf. Theory, vol. 56, no. 5, pp. 2307-2359, May 2010.

[26] W. Yang, G. Durisi, T. Koch, and Y. Polyanskiy, "Quasi-static SIMO fading channels at finite blocklength," in IEEE ISIT, July 2013, pp. $1531-1535$.

[27] — , "Quasi-static multiple-antenna fading channels at finite blocklength," IEEE Trans. Inf. Theory, vol. 60, no. 7, pp. 4232-4265, July 2014. 